\title{
La reconstitution 3D du chevet de Cluny III : clôture de chœur, stalles, pavement, peintures murales et
} vitraux

Juliette Rollier-Hanselmann, Guillaume Schotté, Jérémie Landrieu, Nicolas Poupart et avec la collaboration de Françoise Perrot et Brigitte MauriceChabard 1

\section{(2) OpenEdition \\ 1 Journals}

Édition électronique

URL : https://journals.openedition.org/cem/11962

DOI : $10.4000 /$ cem. 11962

ISSN : 1954-3093

Éditeur

Centre d'études médiévales Saint-Germain d'Auxerre

Édition imprimée

Pagination : 183-201

ISSN : 1623-5770

Référence électronique

Juliette Rollier-Hanselmann, Guillaume Schotté, Jérémie Landrieu, Nicolas Poupart et avec la collaboration de Françoise Perrot et Brigitte Maurice-Chabard 1, " La reconstitution 3D du chevet de Cluny III : clôture de chœur, stalles, pavement, peintures murales et vitraux », Bulletin du centre d'études médiévales d'Auxerre / BUCEMA [En ligne], 15 | 2011, mis en ligne le 22 août 2011, consulté le 22 septembre 2022. URL : http://journals.openedition.org/cem/11962 ; DOI : https://doi.org/10.4000/ cem. 11962

Ce document a été généré automatiquement le 22 septembre 2022.

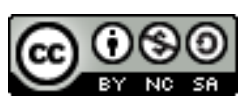

Creative Commons - Attribution - Pas d'Utilisation Commerciale - Partage dans les Mêmes Conditions 4.0 International - CC BY-NC-SA 4.0

https://creativecommons.org/licenses/by-nc-sa/4.0/ 


\title{
La reconstitution 3D du chevet de Cluny III : clôture de chœur, stalles, pavement, peintures murales et vitraux
}

\author{
Juliette Rollier-Hanselmann, Guillaume Schotté, Jérémie Landrieu, Nicolas \\ Poupart et avec la collaboration de Françoise Perrot et Brigitte Maurice- \\ Chabard 1
}

1 Le chevet de Cluny était la partie la plus richement décorée par la présence de sculptures, de peintures, de vitraux, de pavements en marbre et d'objets liturgiques précieux. Une partie de ces éléments nous est parvenue grâce aux fouilles archéologiques et a permis de proposer des hypothèses de restitution. Le parti général était celui de représenter l'église dans son état à la fin $\mathrm{du}_{\mathrm{xv}}^{\mathrm{e}}$ siècle, sans tenir compte des reprises plus tardives. Nous avons tenté de réunir les connaissances actuelles sur chacun des éléments pour proposer une restitution en 3D du chœur ${ }^{2}$, de manière à le présenter sur un écran et dans un film, au cours de la visite sur le site. Il en résulte des images, qui seront amenées à évoluer au fur et à mesure des nouvelles découvertes dans les prochaines années.

\section{Reconstitution de la clôture de chœur}

2 Selon un plan dit anonyme (daté vers 1700) et celui de Giffart (1685 et 1713), une clôture de chœur entourait le sanctuaire et s'avançait jusque dans la onzième travée de la nef centrale, tandis que le plan de Philibert fils (1790) n'indique rien dans cette travée. Le dessin de Lallemand, réalisé vers 1770-1780, illustre un jubé postmédiéval, ce qui n'aide guère à la reconstitution de la clôture romane.

3 C'est l'archéologue américain K.J.Conant qui a retrouvé un grand nombre de fragments appartenant à la clôture du chœur de Cluny III. D'autres éléments proviennent $d u$ fond ancien du musée et de découvertes plus récentes ${ }^{3}$. Plusieurs 
spécialistes ont proposé diverses hypothèses quant à la disposition de ces éléments sculptés. Le chancel à arcatures s'étendait sur dix mètres de long, de manière à séparer la communauté monastique des fidèles. Ces éléments sculptés semblent avoir été mis en place vers $1120-1130$, soit peu après la façade romane (1115-1120) ${ }^{4}$, ce qui permet d'établir des comparaisons avec la sculpture de Vézelay, de Paray-le-Monial, ainsi qu'avec les cathédrales d'Autun (arcades de l'abside), Beaune et Langres.

\section{Clôture basse ou haute ? (fig. 1 et 2)}

4 L'étude de 83 fragments de sculptures a conduit le spécialiste américain E. Armi à proposer l'hypothèse d'un chancel bas ${ }^{5}$, comme cela semble avoir été le cas à SaintDenis vers 1140 et Saint-Étienne de Sens. 78 fragments sont conservés au Musée d'art et d'archéologie de Cluny, d'autres dans des musées américains ou encore dans des collections privées ${ }^{6}$. Les arcatures mesurent environ un mètre de hauteur.

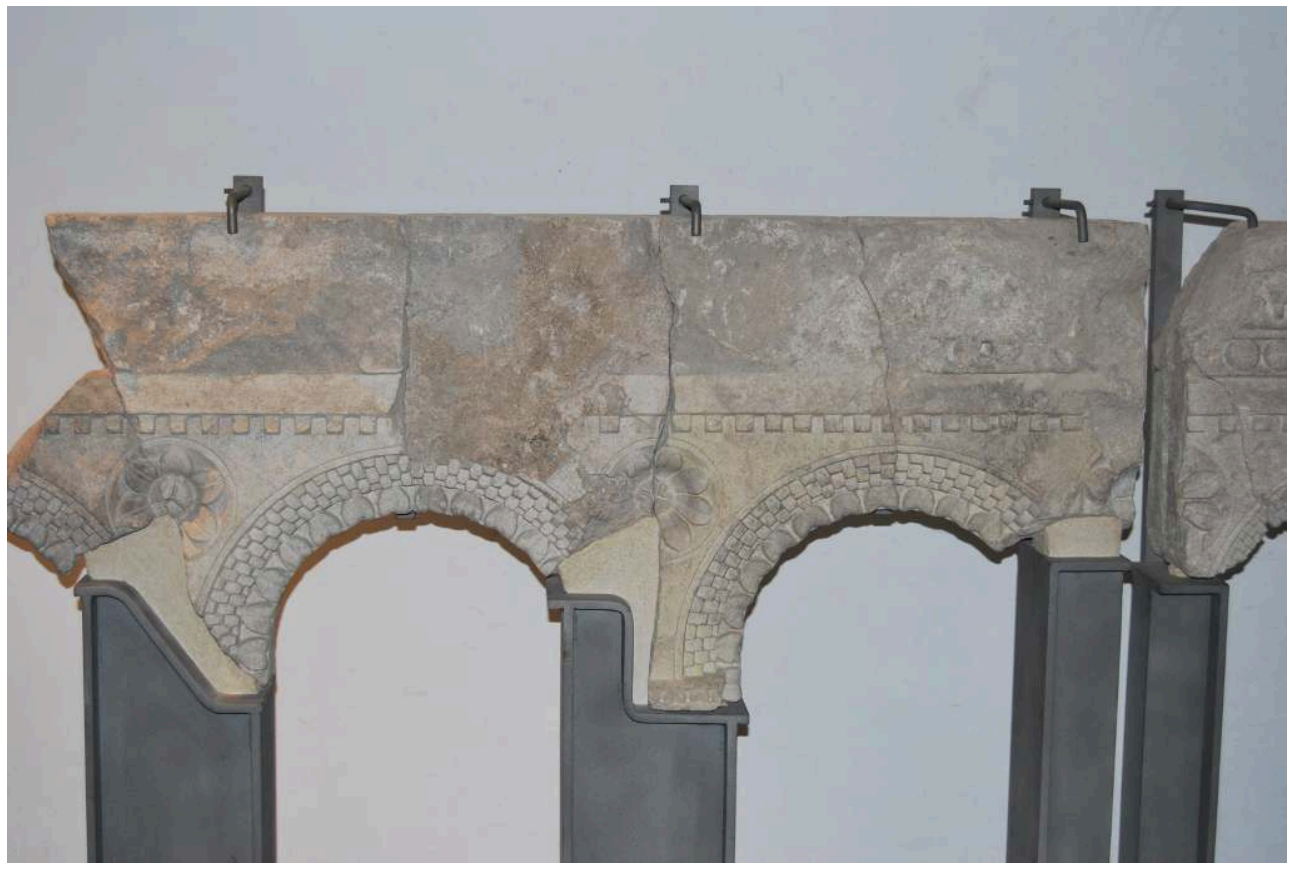

Fig. 1 - Cluny III, clôture de chœur (musée d'art et d'archéologie Ochier, Cluny). 


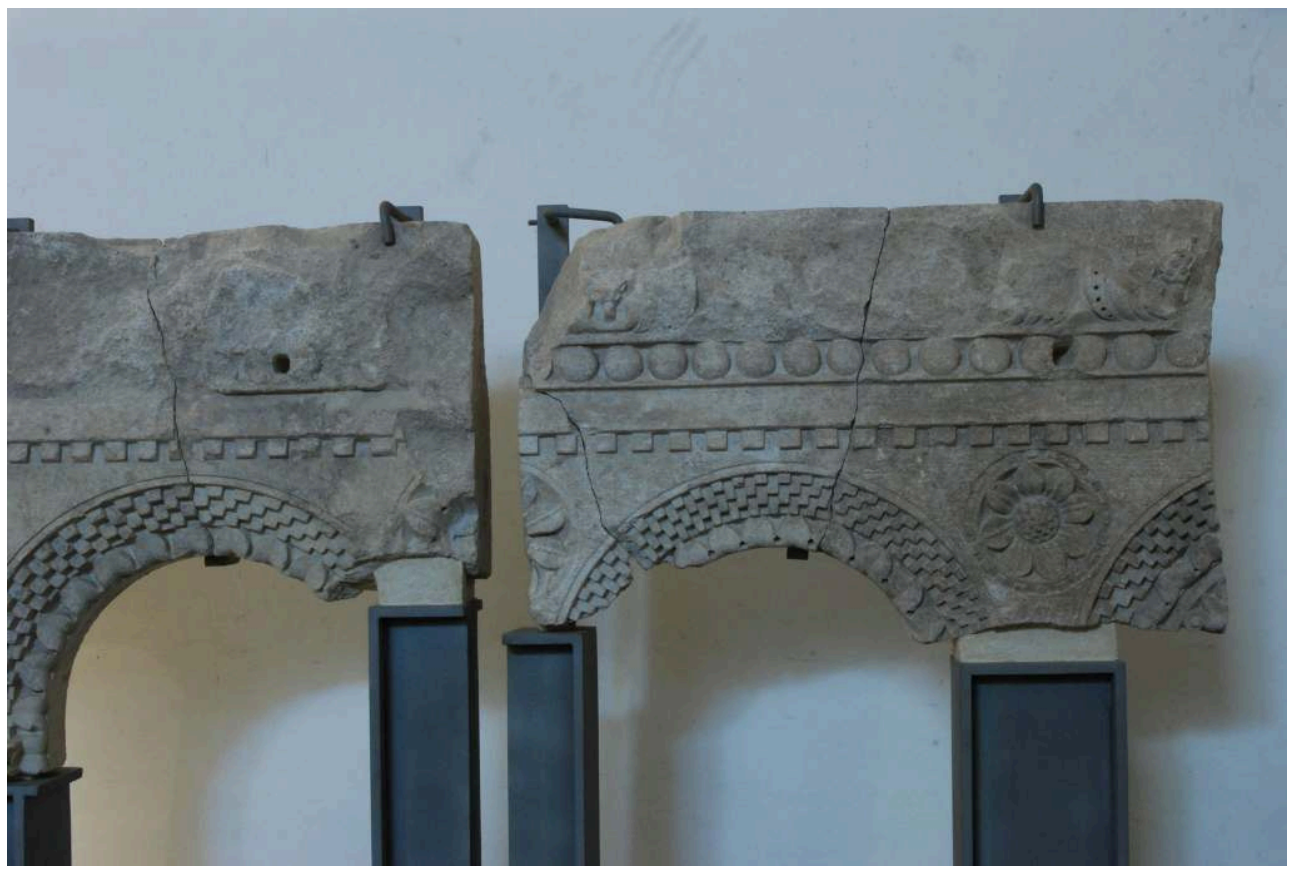

Fig. 2 - Cluny III, clôture de chœur (musée d'art et d'archéologie Ochier, Cluny).

5 Certains arguments militent en faveur d'une clôture basse, ce qui était une solution fréquente avant le $\mathrm{XII}^{\mathrm{e}}$ siècle, notamment dans les grandes basiliques de Rome. La scola cantorum de S. Clemente de Rome (1100-1128), bien que très reprise, illustre ce type de clôture. D'autres exemples du même type subsistent dans les églises romaines - Santi Quatro Coronati et Santa Maria in Cosmedin. À Saint-Denis, vers 1140, l'abbé Suger fit agrandir le chœur et fit poser une structure plus basse, de façon à ne pas gêner la vue vers le sanctuaire.

L'hypothèse d'une clôture haute a été proposée par l'archéologue K. J. Conant ${ }^{7}$, idée reprise par N. Stratford et $\mathrm{D}$. Walsh ${ }^{8}$. Cette structure haute de quatre mètres devait permettre de placer des stalles en gradin pour accueillir près de trois cents moines. Il semblerait que la période située entre la fin $\mathrm{du} \mathrm{XI}^{\mathrm{e}}$ siècle et les années 1200 soit marquée par une évolution de la conception de la barrière de chœur dans les églises d'Occident. Le prieuré clunisien de Souvigny offre un bel exemple au milieu du $\mathrm{XII}^{\mathrm{e}}$ siècle $^{9}$, ainsi que le chœur de Saint-Benoît-sur-Loire. En terre germanique, plusieurs cas existent, notamment à la cathédrale de Naumbourg et à Saint-Michel d'Hildesheim. Le jubé de la Marienkirche de Gelnhausen appartient également à cette évolution. Les clôtures romanes des cathédrales anglaises de Durham et d'Ely et le jubé des chanoines de Vezzolano, près de Turin (daté de 1189), attestent également de l'évolution de ces structures hautes. D'autres observations du même type ont été faites depuis les Pyrénées jusqu'à Saint-Jacques-de-Compostelle, dont la clôture haute était richement sculptée ${ }^{10}$.

7 Selon l'hypothèse de $\mathrm{N}$. Stratford et $\mathrm{D}$. Walsh, la face la plus richement sculptée se trouvait à l'intérieur de la clôture et n'était donc vue que par les moines, la face extérieure étant en grande partie lisse, avec des décors plus simples, probablement peints. Plus tardivement des tentures y furent suspendues.

8 Le vocabulaire ornemental du chancel est très fortement marqué par l'Antiquité. L'alternance d'arcatures ouvertes et aveugles, soutenues par des pilastres cannelés, 
évoque les parties hautes de Paray-le-Monial et Saint-Lazare d'Autun, inspirés par les modèles antiques, telles les deux portes romaines d'Autun.

D'autres fragments conservés dans la Tour du Moulin ont été taillés dans un autre calcaire et selon une technique plus raide, ce qui suggère une date postmédiévale. Les arcatures sont aveugles et correspondent probablement à des restaurations classiques. Les témoignages de Mabillon, Giffart et Dumolin indiquent que des modifications sont intervenues dans le chœur entre 1682 et 1749 . Une reprise complète du chœur a eu lieu en 1781. Une gravure faite vers 1773, d'après le dessin de Lallemand, montre la clôture de chœur avec une petite balustrade à colonnettes et architrave et deux édicules à gauche et à droite ${ }^{11}$. L'analyse de la documentation fait apparaître de multiples modifications du chœur ${ }^{12}$, dont certaines peuvent être dues à des erreurs de transcription entre les manuscrits ou dans le plan de Giffart. Seule une partie de la clôture romane a probablement survécu jusqu'au XVIII ${ }^{\mathrm{e}}$ siècle.

\section{Recherche de polychromies}

10 Malheureusement les plaques présentées au musée de Cluny ne conservent que des restes infimes de polychromies. De nombreux autres fragments ont été conservés dans les réserves très humides du musée, ce qui n'a pas favorisé la préservation des couleurs. Seuls quelques rares fragments donnent une idée approximative de l'ensemble. Un fragment d'arcature (sans numéro) conserve encore une préparation jaune recouverte d'importants restes orange vif, sans que nous puissions distinguer une coloration différente des parties. Un fragment de cannelure présente une préparation jaune et une coloration rouge vif au fond des cannelures, tandis qu'un autre morceau conserve une coloration jaune sur les cannelures se détachant sur un montant latéral rouge ${ }^{13}$. D'autres éléments conservés dans des collections particulières indiquent également une alternance jaune et orange sur les arcatures et du gris sur les pilastres cannelés. Si la coloration très vive de ces éléments de décor ne fait aucun doute, la restitution précise des différentes parties pose problème, si bien que nous l'avons laissée de côté pour l'instant.

\section{La restitution numérique (fig. 3 et 4 )}

La précédente maquette en 3D, réalisée en 2004, a privilégié la restitution d'une clôture basse, selon les hypothèses d'Edson Armi. Pour la nouvelle maquette 2010, nous avons restitué une clôture haute, selon l'hypothèse de K. J. Conant et Neil Stratford/David Walsh. Malgré l'étude des fragments préservés, il est très difficile de définir la configuration précise de la clôture de chœur, qui semble avoir beaucoup évolué selon les époques. Notre restitution se base essentiellement sur le plan Giffart, dans lequel nous constatons que la barrière du chœur ne se positionne pas de la même façon à partir du premier transept - positionnement à l'extérieur des piliers.

12 Le nombre de moines étant estimé à près de trois cents au moment le plus riche de l'abbaye ${ }^{14}$, il convient de disposer les sièges des moines en gradin. Selon K. J. Conant, un haut dossier sert d'appui à trois rangées de stalles, créant ainsi une haute clôture sur les côtés ouest, nord et sud du sanctuaire. Cette hypothèse a été reprise par N. Stratford et D. Walsh. 
13 sélectionné cinq pièces remarquables qui ont été numérisées au laser ${ }^{15}$. Les arcatures en plein cintre s'alternent avec de légères variations dans les motifs. Les grandes plaques sculptées ont été disposées autour du chœur, en insérant des arcades entières entre deux piliers. Lorsque cela n'était pas possible, le motif végétal a été coupé en son milieu. L'épaisseur du mur qui soutient la rangée d'arcatures a été adaptée, de manière à correspondre aux redents des piliers. Le système de montage précis nous échappe, mais des traces indiquent que des agrafes permettaient de maintenir les plaques entre elles.

Aucune information n'existe concernant l'accès vers l'intérieur du sanctuaire. Les fragments qui nous sont parvenus ne fournissent aucune donnée pour établir l'existence d'une entrée axiale. Par analogie avec d'autres exemples (Hildesheim, Compostelle, Serrabonne), nous avons supposé une entrée au centre de la face avant du chancel, s'ouvrant sur un arc en plein cintre. Une grande porte en bois (env. $2 \mathrm{~m}$ ) à deux vantaux permet théoriquement à deux officiants de passer côte à côte. Un entourage de porte a également été proposé, avec des pilastres latéraux (fig. 3).

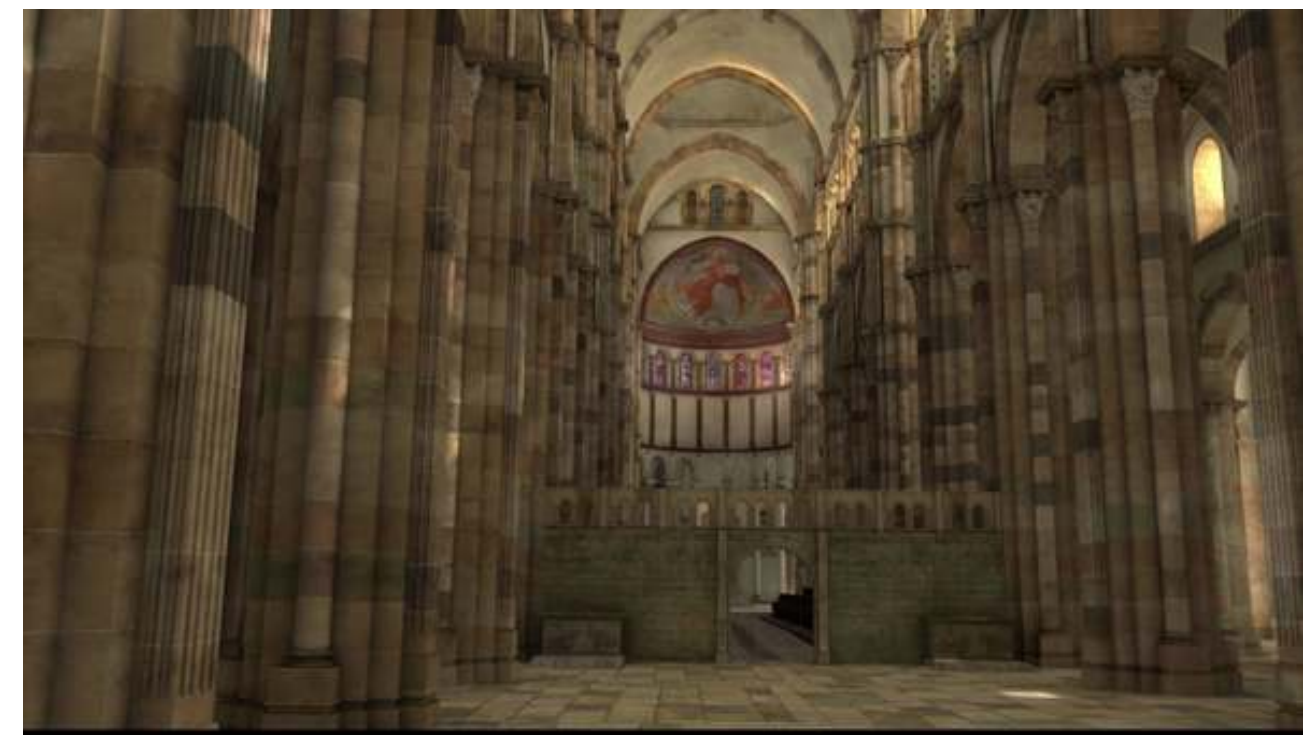

Fig. 3 - Cluny III, restitution virtuelle du chœur issue du film Maior Ecclesia 2010 (Arts et Métiers ParisTech, on-situ, CMN).

Fig. 4 - Cluny III, restitution virtuelle du sanctuaire issue du film Maior Ecclesia 2010 (Arts et Métiers ParisTech, on-situ, CMN). 


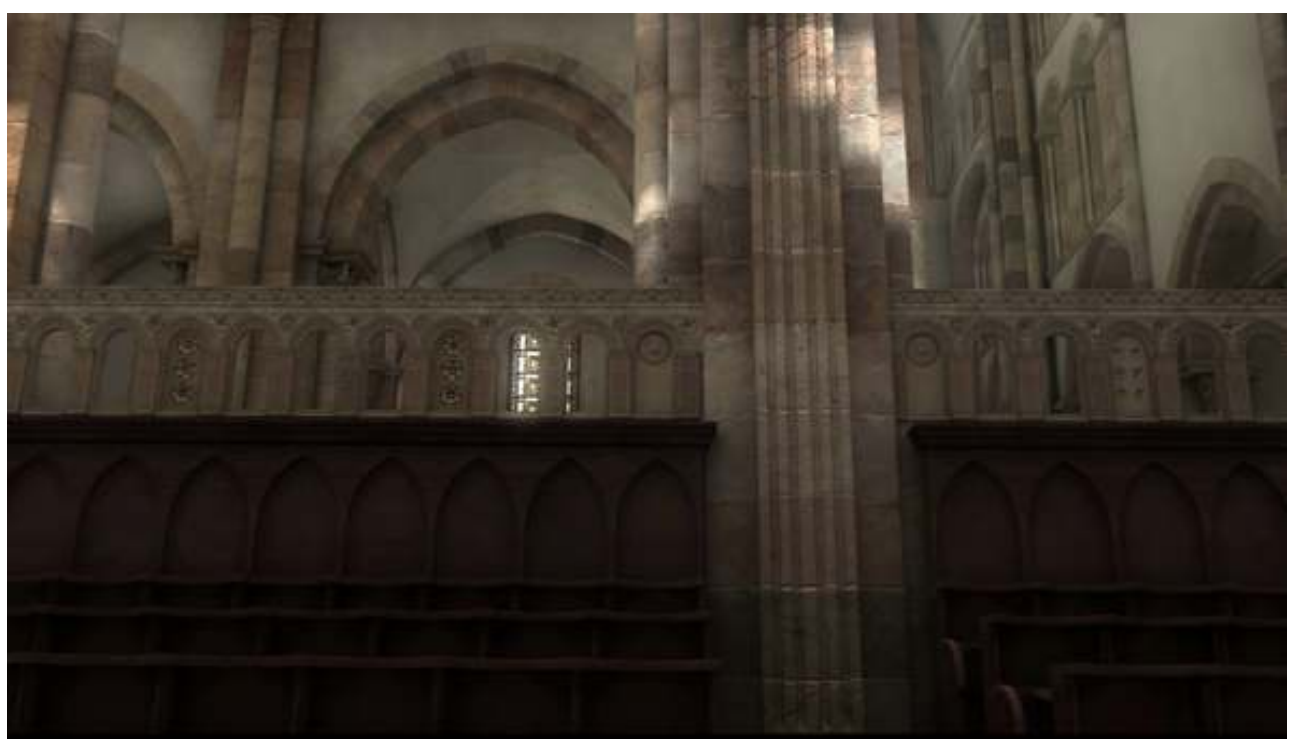

La clôture de chœur devait être munie d'un ou deux ambons, selon un usage connu dès l'époque carolingienne. L'ambon a été détruit lorsque le nouveau chœur a été mis en place avec ses nouvelles stalles en $1781^{16}$. Dumolin situe clairement une chaire à prêcher au niveau du septième pilier de la nef et qui appartient probablement à une époque plus récente.

16 Un élément circulaire a été partiellement reconstitué au musée d'art et d'archéologie de Cluny (fig.5) et d'autres fragments se trouvent dans les réserves du musée qui pourraient appartenir à un ambon ${ }^{17}$. Plusieurs exemples d'ambons médiévaux surélevés sont connus ${ }^{18}$, par les textes, au $\mathrm{x}^{\mathrm{e}}$ siècle dans l'ancienne cathédrale d'Auxerre, ainsi qu'à Liège. Des exemples romans subsistent dans l'ancienne cathédrale de Nice $\left(\mathrm{XI}^{\mathrm{e}}\right.$ siècle) ${ }^{19}$, au prieuré de Malval (Suisse, $\mathrm{XII}^{\mathrm{e}}$ siècle) ${ }^{20}$ et à Notre-Dame de Valère (Sion, fin XII siècle) ${ }^{21}$. L'emplacement précis et la disposition en hauteur de telles structures n'étant pas connu à Cluny, nous avons préféré ne pas les introduire dans la maquette en 3D. D'autres informations concernant ce sujet sont nécessaires, afin de proposer une restitution de ces éléments.

\section{Les stalles en bois (fig. 5)}

17 Les stalles en bois d'époque romane ont disparu et nous n'avons malheureusement plus aucun mobilier de ce type pour le $\mathrm{xII}^{\mathrm{e}}$ siècle ${ }^{22}$. De nombreux fragments de mobilier, attribuables à une balustrade, ont été trouvés par K. J. Conant sur le site de la Chapelle Notre-Dame de l'infirmerie ${ }^{23}$, qu'il estimait provenir de la clôture du chœur XII ${ }^{e}$ siècle. 


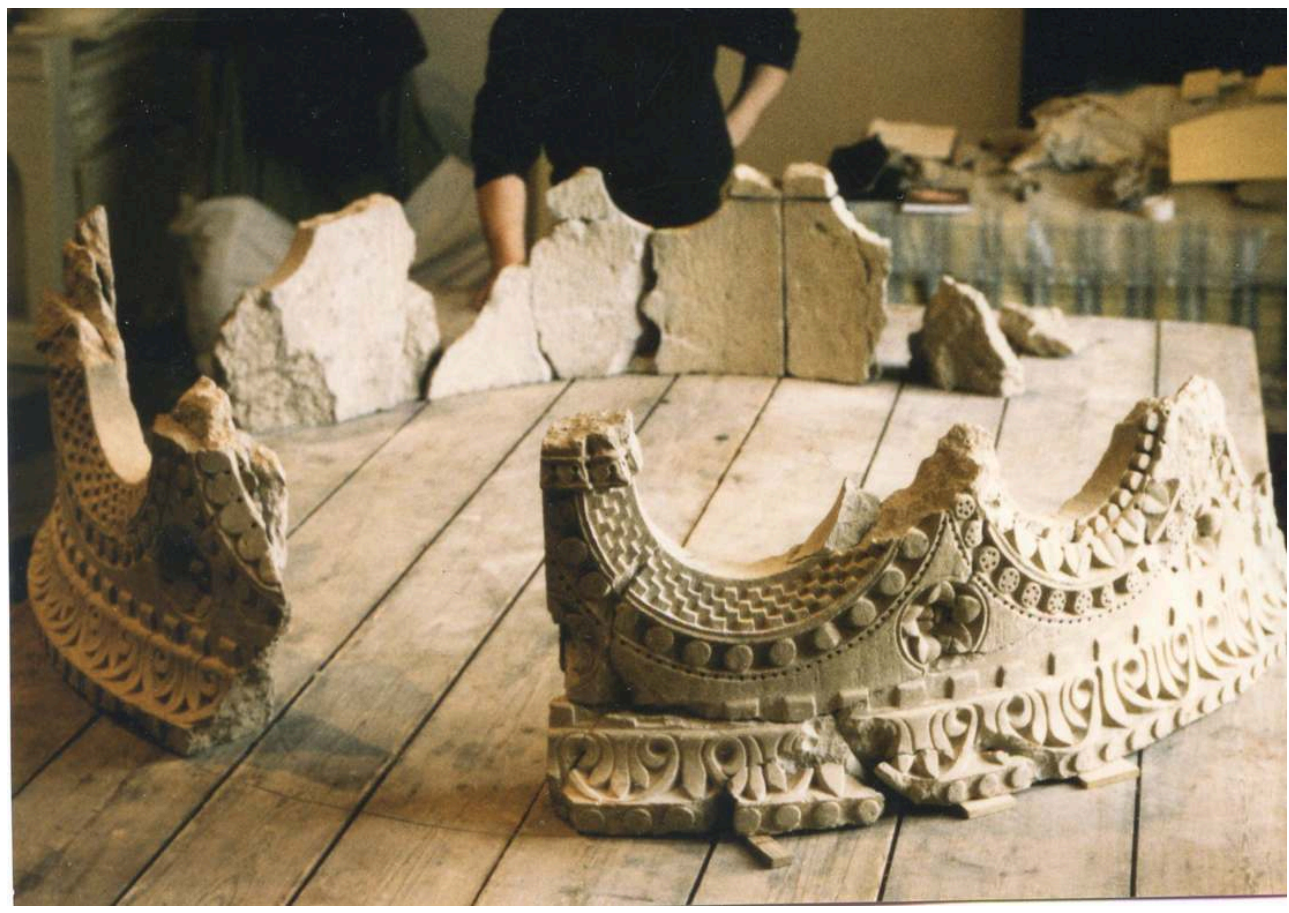

Fig. 5 - Cluny III, élément circulaire en cours de remontage (musée d'art et d'archéologie Ochier, Cluny).

N. Stratford relate les coutumes de Guillaume de Hirsau (vers 1090) ${ }^{24}$, qui décrivent en détail le comportement des moines pendant la liturgie et mentionnent des rangées de stalles et de miséricordes. Il s'agit probablement d'une installation simple, déjà mentionnée dans les coutumes de l'abbaye italienne de Farfa (Liber tramitis), texte daté du deuxième quart du xi ${ }^{e}$ siècle ${ }^{25}$. Par la suite, c'est le statut de Pierre le Vénérable, dès 1146-1147, qui fournit la preuve de l'existence de multiples rangs de stalles à Cluny et d'un système de charnières. Gervais de Canterbury mentionne également les stalles comme un facteur déterminant lors du grand incendie de la cathédrale en $1174{ }^{26}$. Étant donné le nombre important de moines présents à Cluny, N. Stratford et D. Walsh supposent une structure en bois de grandes dimensions, telle que nous en connaissons à partir du XIII ${ }^{\mathrm{e}}$ siècle.

Pour la restitution virtuelle, nous avons choisi un modèle de siège simple et massif, en les plaçant sur trois rangées, posées sur des gradins en bois. Des escaliers d'accès entre les stalles et des couloirs d'accès ont été installés pour permettre une circulation aisée. Pour la dernière rangée, un dossier en arc brisé, de type gothique, permet d'appuyer le mobilier contre la clôture. Cette disposition permet de placer 246 moines dans le chœur.

Quant aux stalles XVIII ${ }^{\mathrm{e}}$ siècle qui subsistent de l'abbaye de Cluny, nous ne les avons pas intégrées dans notre maquette, dont le contenu comporte les données médiévales jusqu'à la fin du Xve siècle. Selon L. Niepce ${ }^{27}$ (1881), ces stalles de 1781 furent transportées à Lyon en 1802 pour être installées dans la cathédrale Saint-Jean et ensuite dans le petit séminaire. 


\section{Hypothèse de reconstitution du pavement de Cluny III (fig. 6 et 7 )}

\section{Les motifs}

Les fouilles de K. J. Conant, dans les années 1931-1932, ont conduit à la découverte de fragments de mosaïque de pavement provenant du chœur de Cluny III. Plusieurs plans de fouilles, ainsi que des dessins des fragments, accompagnent les commentaires des carnets de fouilles. Divers secteurs ont livré des morceaux ${ }^{28}$, avec cependant deux sondages plus riches dans le chœur, entre les deux autels, et dans une moindre mesure à l'extérieur du chœur. L'ensemble de ces secteurs a livré plus de 150 fragments de pavement à incrustations de pierres (opus sectile) et en petites tesselles (opus tessellatum), ainsi qu'un élément à incrustations de verre. Une étude précise du matériel a été réalisée dans le cadre du «Corpus de la sculpture de Cluny ${ }^{29}$.

Le répertoire des motifs est varié, avec divers éléments réalisés en opus sectile, notamment les rosaces ou fleurs blanches sur fond noir, les peltes et les losanges. Les fleurs à doubles pétales s'inscrivent dans un encadrement blanc ; quatre grands pétales biconvexes alternent avec quatre petits pétales lancéolés. Dans certains cas, il ne subsiste que l'empreinte en négatif, dans l'enduit. De petites rosaces à quatre pétales blancs ont également été posées sur fond de tesselles noires, l'ensemble du motif étant compris dans un carré d'environ $15 \mathrm{~cm}$ de côté. D'autres éléments, qui combinent les petites tesselles de mosaïques et l'opus sectile, appartiennent probablement à des motifs géométriques plus grands, tels que les cercles sécants. Dans ce cas, les tesselles permettent d'épouser les motifs arrondis.

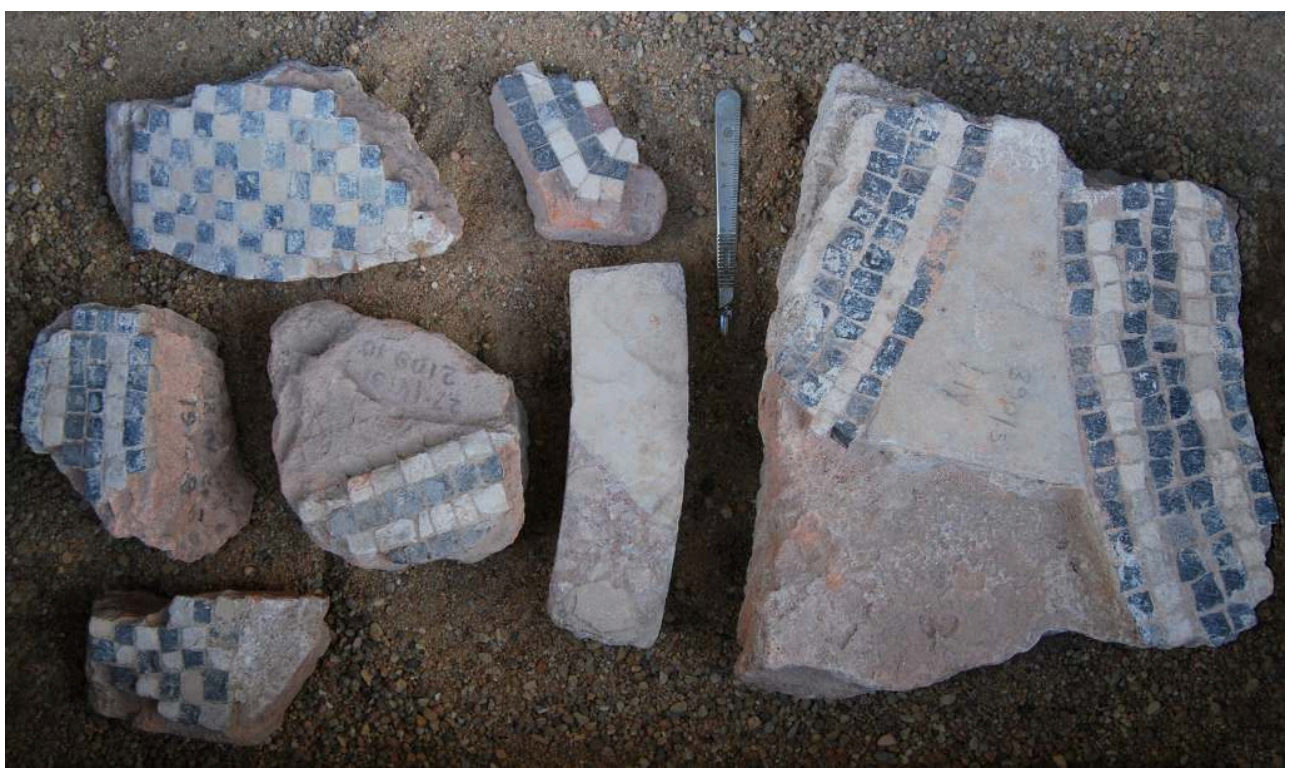

Fig. 6 - Cluny III, fragments de pavement du chœur (musée d'art et d'archéologie Ochier, Cluny). Fig. 7 - Cluny III, fragments de pavement du chœur (musée d'art et d'archéologie Ochier, Cluny). 


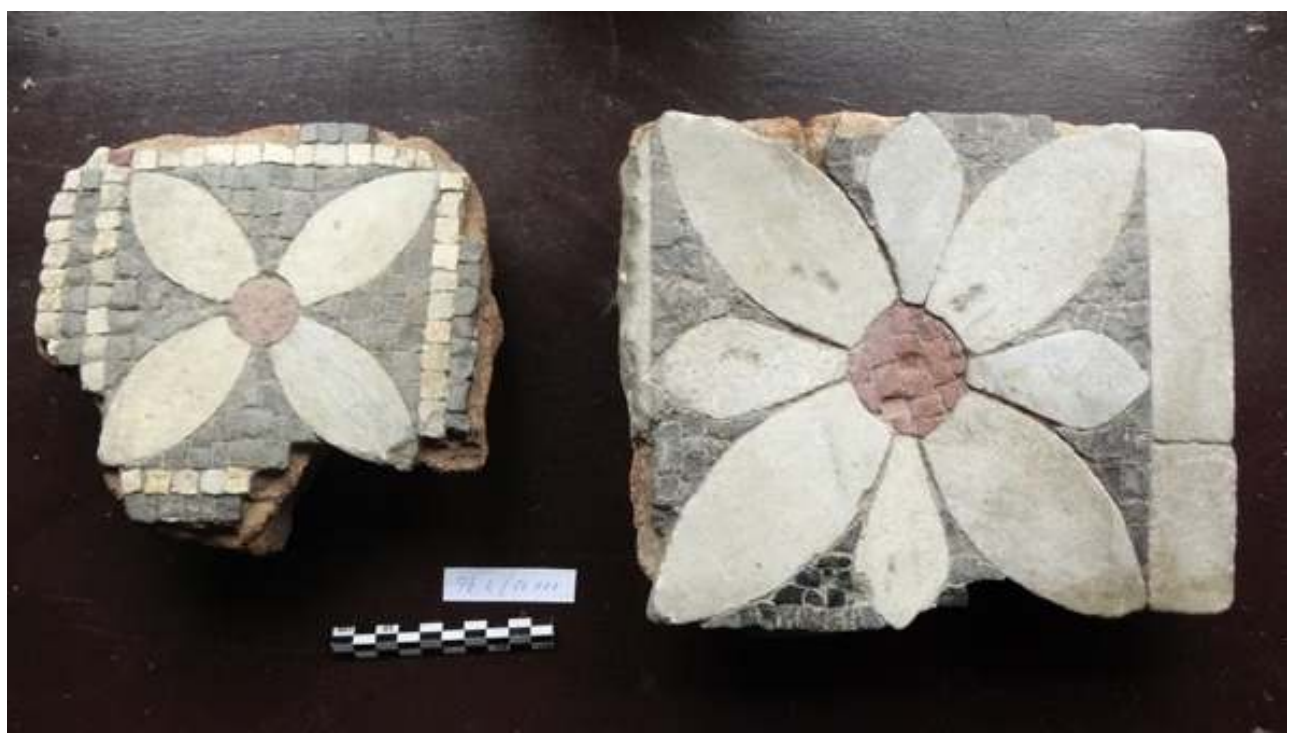

\section{Les matériaux} d'un riche pavement au niveau du chœur : «La portion du sanctuaire qui s'étend depuis le grand autel jusqu'au chœur est une mosaïque travaillée en roses, en étoiles, en échiquier exprimés avec beaucoup de délicatesse... De la mosaïque à l'autel matutinal, ce qui achève de donner l'étendue du sanctuaire, règne un parquet à compartiments formés par des losanges de marbre noir et blanc. » éléments provenant de Bourgogne (calcaire rouge, schiste), mais également des produits d'importation, comme les marbres blancs et blancs veinés - Alpes, Pyrénées, bassin méditerranéen -, le cipolin vert (Grèce), la brèche coralline (Turquie), du porphyre vert de Laconie (Grèce). Les matériaux sont issus de remplois à partir de monuments antiques de la région lyonnaise, de Chalon-sur-Saône et d'Autun. Les couleurs sont habituelles pour l'époque, avec des dominantes noire, blanc, rouge foncé, et quelques rares éléments verts. Dans certains cas, les grandes dalles circulaires de marbre pouvaient être retaillées dans des colonnes antiques ${ }^{32}$.

répertoire décoratif indique que le pavement de la grande église de Cluny III devait ressembler à des exemples d'Italie centrale et du Nord ${ }^{33}$. Les pavements romains - San Lorenzo fuori le mura, San Clemente, Saint-Jean du Latran, Sainte-Praxède ${ }^{34}$-, divers exemples du Latium (Farfa, Castel Sant'Elia ${ }^{35}$ ) et de France (Saint-Benoît-sur-Loire) permettent d'établir un certain nombre de comparaisons et de proposer une hypothèse de restitution pour Cluny.

\section{La disposition}

La disposition du pavement du chœur étant tributaire du mobilier, nous avons d'abord tenté de reconstituer l'emprise des stalles, de manière à mieux estimer la surface disponible pour l'allée centrale. Parmi les fragments retrouvés dans les fouilles de K. J. Conant, certains éléments courbes permettaient de prolonger les tracés et de reconstituer les grands cercles sécants d'un mètre de diamètre, qui forment une sorte

Bulletin du centre d'études médiévales d'Auxerre | BUCEMA, 15 | 2011 
de tapis monumental menant à l'autel majeur, long de 30 mètres. L'espace compris entre les stalles était large $(3,73 \mathrm{~m})$, ce qui permet de compléter le grand tapis de cercles par des panneaux latéraux variés. De part et d'autre du tapis central, nous avons essayé de répartir les autres motifs répertoriés, soit sous forme de bandes longeant le tapis central (motif de peltes), soit sous forme de tapis rectangulaires motifs des rosaces et de peltes.

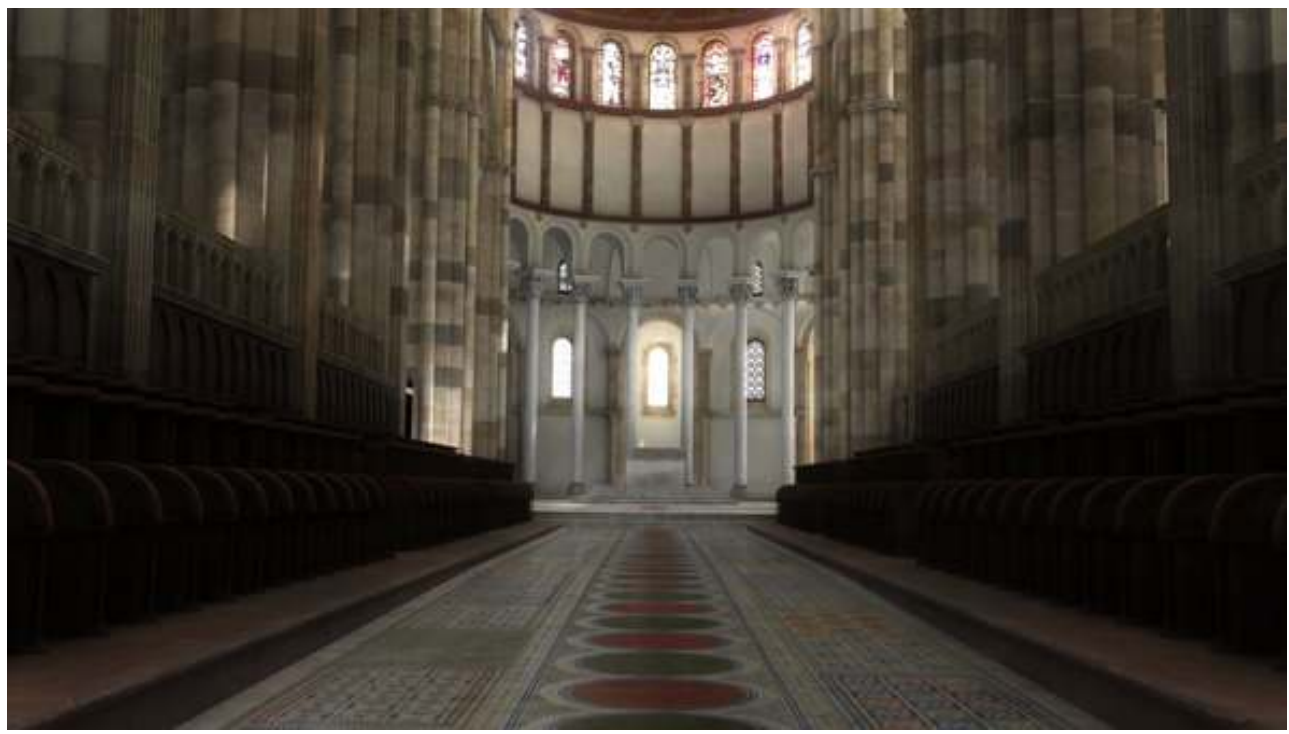

Fig. 8 - Cluny III, reconstitution virtuelle du pavement central du sanctuaire issue du film Maior Ecclesia 2010 (Arts et Métiers ParisTech, on-situ, CMN)

Un fragment très intéressant comporte l'entrelacement de plusieurs courbes, dont la prolongation aboutit à l'intersection de quatre cercles sécants, que nous avons placés devant l'autel majeur, en correspondance avec les bras du grand transept.

Le cheminement des moines sur le tapis central, menant à l'autel majeur, mesure 45 mètres jusqu'à l'emmarchement de la grande abside, ce qui laissait toute latitude pour l'accomplissement des chants et des rites liturgiques.

Entre les deux autels, les découvertes archéologiques semblent indiquer un autre rythme dans les pavements, ce que nous avons cherché à restituer dans notre maquette. C'est le modèle de Castel S. Elia, site clunisien au nord de Rome, qui a servi de base pour notre travail. Il semblerait que le pavement de ce sanctuaire ait été mis en place avant 1120-1121.

30 La reconstitution du pavement dans le sanctuaire représente une surface totale de $390 \mathrm{~m}^{2}$. L'aspect légèrement brillant des marbres et des calcaires utilisés rendait cet ensemble extrêmement riche et devait refléter la lumière d'une façon bien particulière.

\section{Le décor peint de la grande abside (fig. 9 et 10)}

\section{Les fragments d'enduits peints}

31 K. J. Conant a mis au jour des dizaines de fragments d'enduits peints en divers endroits du domaine abbatial, notamment à proximité de l'ancienne abside de Cluny II, à l'intérieur de Cluny III, ainsi que dans l'ancien cloître ${ }^{36}$. Plus récemment, lors des fouilles réalisées en 1989 à proximité de l'avant-nef, les archéologues ont également 
découvert une couche de remblai riche en enduits peints ${ }^{37}$, qui présentent des analogies stylistiques et techniques frappantes avec le matériel de K. J. Conant.

Divers fragments de carnations, de vêtements - plis roses, verts, jaunes -, de bordures et d'autres éléments permettent d'affirmer que des peintures du même type que celles de la Chapelle-des-Moines ornaient Cluny III. La palette des peintres de Cluny et de Berzé est identique - blanc de chaux, ocres jaunes et rouges, minium, vermillon, terre verte, bleu de lapis-lazuli, noir de charbon -, tout comme la technique picturale mixte base à fresque, finitions à la chaux, stratigraphies ${ }^{38}$.

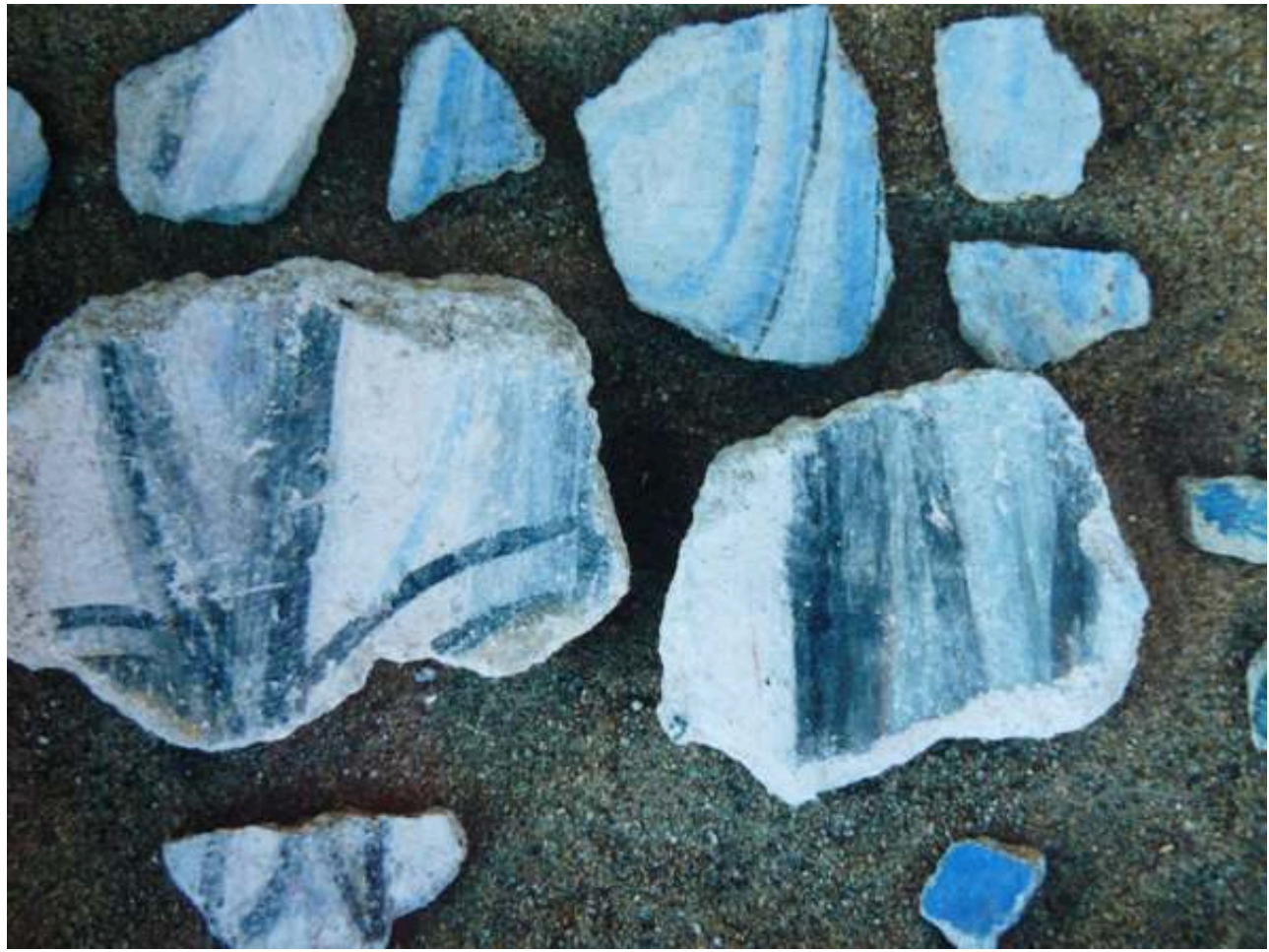

Fig. 9 - Cluny III, fragments d'enduits peints provenant de la grande abside (musée d'art et d'archéologie Ochier, Cluny).

\section{La documentation iconographique}

Dans son Corpus de la sculpture de Cluny, Neil Stratford a rassemblé les documents concernant l'abside principale de Cluny III ${ }^{39}$. Le plus ancien texte date du 21 juin 1726 lorsque dom Urbain Plancher décrit la peinture en ces termes: «il y a un Sauveur représenté à la manière du onze et douzième siècle ». Malheureusement, le dessin qu'il a fait réaliser par un dessinateur a disparu ${ }^{40}$. Vers 1749 , le docteur Dumolin écrit une histoire de Cluny, en mentionnant l'abside ainsi : « une voûte en demi-cul-de-four... est décorée de plusieurs figures en plate peinture; celle du centre, renfermée dans un ovale, imite le Père éternel, et les autres placées à la circonférence expriment le symbole des quatre évangélistes et des chœurs qui forment la cour céleste ».

dessin sommaire de Guyot ${ }^{41}$, publié en 1840 par Alexandre Lenoir, est accompagné de la description suivante : «La coupole peinte à l'eau d'œuf selon la manière du temps est rehaussée d'or et les figures qui y sont représentées, dans la proportion de 10 pieds, se détachent sur un fond d'or chargé d'ornements qui imitent la mosaïque. Ce tableau unique en Europe a conservé toute sa fraîcheur et toute sa vivacité ; il représente le 
Christ assis sur des nuages, appuyé sur l'Évangile et entouré des animaux de l'Apocalypse... ${ }^{42}$. " Selon la description de M. P. Lorain (1839), il y avait aussi un agneau aux pieds du Christ ${ }^{43}$.

K. J. Conant, qui publie le document de Lenoir, propose d'y voir une restauration datant de 1750 , ce qui nous paraît une hypothèse intéressante ${ }^{44}$. En effet, une campagne générale de travaux a lieu cette année-là et la toiture de l'abside est remplacée par une nouvelle couverture conique. Cette information, qui indique l'état de délabrement du bâtiment, sous-entend probablement une restauration importante des peintures de l'abside. En effet, l'image de Lenoir ressemble plus à une copie moderne qu'à une image médiévale. Le dessin montre un Christ dont le corps est assez ample. Les proportions et les gestes sont reproduits avec beaucoup de gaucherie. Il faut remarquer l'absence de nimbe et de mandorle, qui est surprenante. L'articulation du drapé et la position des symboles des évangélistes n'ont rien de médiéval. L'allure générale indique probablement qu'il s'agit d'une peinture moderne.

Un autre document du XIX siècle apporte des informations complémentaires. Il s'agit d'une lettre de M. Furtin, maire de Cluny, datée de $1811^{45}$, qui semble décrire la peinture du cul-de-four de façon plus fidèle : «Le fond de l'ellipse est peint en jaune d'ocre, parsemé d'étoiles d'or ou dorée, les gloires des Saints sont de minium, les draperies sont bleues rehaussées de rouge, la bordure de l'ellipse et le siège du Sauveur sont en mosaïque, ainsi que tous les ornements qui font partie de cette peinture. » La description parait assez réaliste, le principe des incrustations dorées dans le fond des mandorles étant un principe bien connu dans d'autres absides de Bourgogne. Même si les éléments rapportés dans la mandorle du Christ à Berzé-la-Ville ${ }^{46}$ et Nevers (boules de verre) ${ }^{47}$ ne sont pas conservés, des imitations peintes en forme d'étoiles sont visibles dans d'autres sites (Tournus, Chalivoy-Milon), qui indiquent leur présence.

L'interprétation de la documentation reste cependant incertaine. Étant donné que des dorures sont présentes sur le portail roman de Cluny III ${ }^{48}$, nous supposons que le prestige et l'importance de Cluny justifiaient ce type de richesse décorative dans les peintures murales, ce qui posait un certain nombre de problèmes techniques. Dans les rares exemples de dorures monumentales qui subsistent, les feuilles d'or et d'argent se limitent à quelques détails bien précis: auréoles, rangs de perles et galons de vêtements ${ }^{49}$.

Nous terminerons par la gravure d'Émile Sagot, publiée par Fernand Mercier en 1931, qui montre le traditionnel Christ en Majesté entouré du tétramorphe ${ }^{50}$. Le document présente de grandes figures en pied sur les écoinçons selon un schéma paléochrétien. Il est difficile de dire si cette gravure correspond à ce que son auteur a vu réellement ou s'il s'agit d'une interprétation.

\section{La restitution}

Lorsque K. J. Conant a fouillé le chevet, il a retrouvé un certain nombre de fragments d'enduits peints appartenant à plusieurs périodes ${ }^{51}$. Les fragments appartenant à l'époque romane permettent de dire que l'abside était certainement peinte et qu'elle présentait les caractéristiques de la grande peinture clunisienne à son apogée, telle que nous la connaissons dans la Chapelle-des-Moines à Berzé-la-Ville. La documentation semble attester de reprises et de repeints, ce que nous constatons également sur les fragments, dont certains comportent plusieurs couches picturales. 


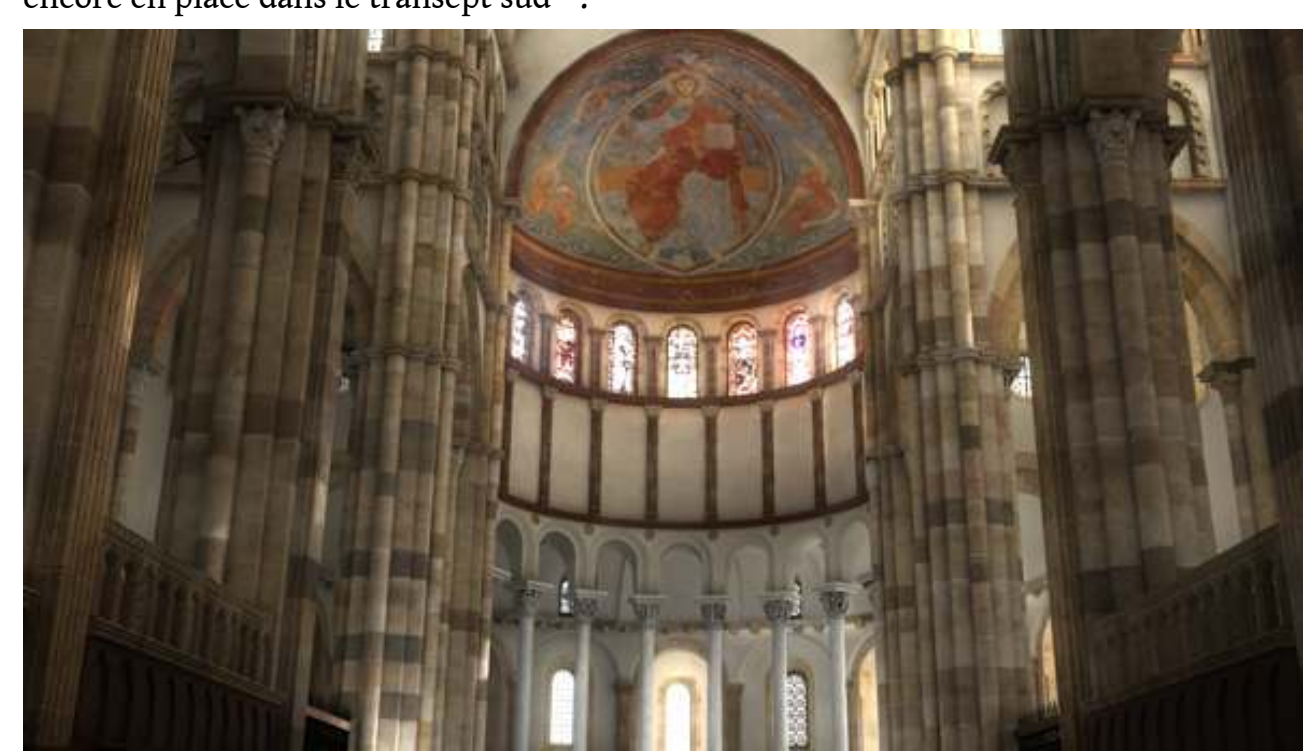

Fig. 10 - Cluny III, reconstitution virtuelle de l'abside centrale issue du film Maior Ecclesia 2010 (Arts et Métiers ParisTech, on-situ, CMN).

\section{Les vitraux de l'abbatiale} était éclairée par de nombreux vitraux, sans que nous puissions déterminer s'ils appartenaient à l'époque romane, gothique ou aux réfections ultérieures: «l'église est percée de trois cents et un vitraux ${ }^{53} »$.L'auteur parle plusieurs fois de vitraux, dans l'avant-nef et dans les nefs, sans donner de détails.

Lors des fouilles du bras sud du transept en $19944^{54}, 780$ fragments de verre ont été mis au jour, parmi lesquels figurent des vitraux colorés médiévaux et des éléments de lampes. La stratigraphie des fouilles a permis de reconstituer la chronologie du chantier, avec des couches appartenant aux niveaux de construction et de destruction 
de Cluny III. Des fragments de vitraux authentiquement romans attestent ainsi de la présence d'ouvertures colorées et historiées.Leur conditionnement en milieu humide n'a guère favorisé leur bonne conservation, ce qui rend leur étude d'autant plus difficile. La taille des fragments est variable, allant de quelques millimètres jusqu'à 5 ou $6 \mathrm{~cm}$ pour les plus importants.

Les verres romans sont généralement reconnaissables à leur épaisseur (3-4 mm) et leur état de conservation très oxydé et très assombri. Parmi les 150 fragments recensés, nous distinguons des éléments verdâtres, des rouges intenses, un bleu profond et des grisailles peintes en rouge-brun ou noir, selon les éléments. Un certain nombre de fragments sont devenus noirs et opaques, avec une grisaille visible en surface, mais l'état d'oxydation ne permet plus de connaître la couleur d'origine du verre. Dans certains cas, la matrice intérieure est de teinte verdâtre, avec les surfaces extérieures noires ou comportant des grisailles. Dans d'autres cas, le verre est devenu complètement opaque et noir. La fragilité du matériau rend leur nettoyage difficile et certaines grisailles sont devenues sensibles à l'eau.

L'état lacunaire des grisailles ne permet plus d'établir un répertoire de motifs, mais nous distinguons clairement des éléments de plis qui pourraient appartenir à des vêtements ${ }^{55}$, ce qui confirme la présence de décors historiés. Les couleurs des grisailles sont variées : le gris-blanc, le rouge et le noir. Les coups de pinceaux sont tantôt fluides, laissant une matière plane, ou plus liquides, créant des épaisseurs de matière. Deux techniques picturales ont été utilisées : l'une, où la grisaille sert à marquer les détails des plis en rouge-brun sur un verre clair, l'autre, selon laquelle la grisaille noire sert à peindre les ombres, les plis apparaissant en réserve.

Le grand transept sud conserve également deux arcatures en partie haute, dont les enduits romans sont ornés de faux-vitraux ${ }^{56}$, peints en motif en résille. Ces éléments sous-entendent probablement une alternance entre vitraux incolores, aniconiques et les parties colorées et historiées, probablement réservées aux principaux pôles liturgiques. D'autres exemples de vitraux neutres sont connus, à Bavay, où une baie circulaire est peinte d'un quadrillage, à San Vincenzo al Volturno (Italie centrale), ainsi que dans divers sites médiévaux ${ }^{57}$.

Les fouilles ont également apporté de nombreux vitraux incolores, dont certains sont médiévaux, d'autres plus tardifs. Quant à la chapelle Jean de Bourbon ( $\mathrm{xv}^{\mathrm{e}}$ siècle), les fragments indiquent des vitraux historiés, que nous laissons de côté ici. Les analyses de laboratoire permettent d'établir des comparaisons intéressantes avec ceux de la basilique de Paray-le-Monial ${ }^{58}$.

\section{La restitution des vitraux dans la maquette virtuelle}

L'étude archéologique et historiographique des vitraux s'est avérée complexe. Elle nous a permis de cerner la problématique d'une manière globale sur divers sites clunisiens et dans un contexte français plus large. Afin de suggérer la présence de vitraux médiévaux colorés et historiés dans la grande abbatiale, nous avons procédé à une sélection d'un certain nombre de verrières correspondant à la période envisagée. Il nous paraissait excessif de prévoir des vitraux colorés et historiés dans toutes les parties de l'édifice, d'autant plus que des faux-vitraux sont présents dans la partie haute du grand transept 
sud. Nous avons alors opté pour une solution intermédiaire, avec des éléments décorés dans le chevet et des parties plus sobres le long des nefs.

Il s'agissait, tout d'abord, de choisir des ferrures types pour le xiIe siècle, à savoir des éléments rectilignes, selon le modèle de Chartres - façade occidentale, vitrail de l'enfance du Christ. Nous devions, ensuite, trouver des images qui puissent être insérées dans un édifice consacré en 1095 et terminé vers 1120 pour les parties romanes. La crucifixion de la cathédrale de Poitiers (vers 1165-1170)nous a paru un exemple qui pouvait s'insérer dans la baie axiale de Cluny III ${ }^{59}$. L'exemple de la cathédrale du Mans (1130-1140), avec de grandes figures élancées, en position presque dansantes, pouvait s'insérer dans la production clunisienne de l'époque ${ }^{60}$. La restitution des verrières du chœur de Cluny provient donc d'images de ces édifices.

51 La présence de trois cents vitraux, comme l'indique le témoignage de Benoît Dumolin au milieu du XVII ${ }^{e}$ siècle, sous-entend la présence d'un important atelier sur place et dont nous n'avons aucune trace. Étant donné la présence de deux ouvertures bouchées et peintes en faux vitrail dans la partie haute du grand transept sud, nous étions en mesure de supposer que d'autres parties étaient éclairées de façon plus sobre. Pour les ouvertures des cinq nefs, nous avons opté pour des vitraux aniconiques, légèrement teintés, selon la palette observée sur les fragments archéologiques étudiés. Qu'il soit roman ou gothique, le verre médiéval retrouvé sur le site n'est jamais blanc, ni transparent, mais plutôt de teinte verdâtre et translucide. Afin d'éviter l'aspect trop répétitif des verrières, une alternance de motifs simples a été intégrée dans la restitution virtuelle, selon le modèle des grisailles d'Orbay. Une difficulté résidait dans l'application informatique, car la présence de vitraux colorés réduit sensiblement la lumière. Dans les nefs il a fallu alterner avec des vitraux partiellement transparents pour remédier à cet inconvénient.

En conclusion, la restitution virtuelle d'un édifice tel que la grande abbatiale de Cluny III soulève de nombreuses questions et permet de réfléchir de manière renouvelée à la production des décors à la fin $\mathrm{du} \mathrm{XI}^{\mathrm{e}}$ et au début du XII ${ }^{\mathrm{e}}$ siècle. L'imagerie virtuelle permet de montrer des hypothèses de travail, qui seront amenées à évoluer au fur et à mesure des recherches et des fouilles sur le terrain.

\section{NOTES}

1. G. Schotté et J. Landrieu, ingénieurs 3D, équipe Gunzo, Arts et Métiers ParisTech, Cluny; N. Poupart, étudiant «Fontanet», Arts et Métiers ParisTech, Cluny; F. Perrot, directrice de recherche au CNRS ; B. Maurice-Chabard, conservatrice des musées d'Autun.

2. Projet de restitution de l'abbaye de Cluny III pour le $1100^{\mathrm{e}}$ anniversaire de l'abbaye, Christian Père (dir.), maître de conférences, Arts et Métiers ParisTech, Institut Image, CNRS 5158.

3. B. MAURICE, Cluny III, la Maior Ecclesia, Cluny, 1988, p. 98-100.

4. .E. ARMI, "The Choir Screen of Cluny III", Art Bulletin (1984), p. 565 ; E. ARMI, Masons and Sculptors in Romanesque Burgundy: The New Aesthetic of Cluny III, University Park, 1983, chap. V, App. 4. 
5. E. ARMI, « The Choir Screen... ", op. cit., 1984, p. 556-573.

6. Un morceau se trouve à la Smart Gallery de l'université de Chicago. Les morceaux plus élaborés et de taille plus importante sont au musée des Cloisters du Metropolitan Museum of Art de New York.

7. K. J. CONANT, Cluny, les églises et la maison du chef d'ordre, 1968, p. 31, note 47.

8. N. S TRATFORD, B. MAURICE-CHABARD, D. WALSH etalii, Corpus de la sculpture de Cluny. Les parties orientales de la Grande Église Cluny III, Paris, 2011.

9. N. STRATFORD, La frise monumentale romane de Souvigny, Souvigny, 2002.

10. E. CARRERo SANTAMARIA, "Centro y periferia en la ordenación de espacios litúrgicos: las estructuras corales », Hortus artium medievalium, 14 (2008), p. 159-179.

11. E. ERLANDE-BRANDENBOURG, «Iconographie de Cluny III ", Bulletin monumental, 126 (1968), p. 292.

12. E. ARMI, « The Choir Screen... ", op. cit., p. 556-573.

13. Fragment $n^{\circ} 89,1.52 \mathrm{~A}$, panier 4 .

14. K. J. Conant, Cluny..., op. cit., p. 31.

15. Il s'agit des grands éléments conservés au musée d'art et d'archéologie, ainsi qu'une corniche se trouvant dans la réserve 2 .

16. . E. ARMI, « The Choir Screen... ", op. cit., p. 560, note 28.

17. Parmi les pièces présentées au musée de Cluny, nous observons quelques infimes restes colorés rouge vif.

18. E. ARMI, « The Choir Screen... », op. cit., p. 562.

19. J. THIRION, «L'ancienne cathédrale de Nice et sa clôture de chœur du $\mathrm{XI}^{\mathrm{e}}$ siècle d'après les découvertes récentes », Bulletin de la Société nationale des antiquaires de France (1966), p. 32-40.

20. L. BLONDEL, « Un prieuré inconnu : le temple de Malval », Genava, 12 (1964), p. 107-121.

21. Kunstdenkmäler in der Schweiz. Ein Bildhandbuch, éd. R. ноотZ, Munich, 1970, 11, pl. 321.

22. C. Charles, Stalles sculptées du Xve siècle: Genève et le duché de Savoie, Paris, 1999. Nous remercions $C$. Charles de nous avoir aidé dans le choix des stalles pour la maquette de Cluny III.

23. Pits LIV, LVIII.

24. N. Stratford cite le passage des coutumes de Hirsau. Éd. PL, 150, col. 961-963 (Sancti Wilhelmi Constitutiones Hirsaugienses seu Gergenbacenses, Liber I, cap. XXIX).

25. Corpus Consuetudinum Monasticarum, XII-2, p. 66.

26. N. STRATFORD et alii, Corpus de la sculpture de Cluny..., op. cit.; C. TRACY, English Gothic Choir Stalls, 1200-1400, Woodbridge, 1987.

27. L. NIEPCE, Archéologie lyonnaise, Lyon, 1881.

28. B. MAURICE, «Les pavements du chœur de Cluny III ", in Le gouvernement d'Hugues de Semur à Cluny, Cluny, 1988, p. 363-383.

29. Étude de B. Maurice-Chabard partiellement publiée dans N. STRATFORD (dir.), Cluny 910-2010. Onze siècles de rayonnement, Paris, 2010; B. MAURICE-CHABARD, «Fussbodenplatte mit Rosettenmotiv ", in Canossa 1077-Erschütterung der Welt, t. 2, Paderborn, 2006, p. 86-87.

30. B. DUMolin, Description historique et topographique de la ville, abbaye et banlieue de Cluny, Cluny, 1749-1798 [musée Ochier, ms. 71].

31. Analyses effectuées par A. Blanc dans le cadre de l'ouvrage Corpus de la sculpture de Cluny..., op. cit.

32. P.C.ClAusSen, Magistri Doctissimi Romani. Die Römischen Marmorkünstler des Mittelalters, Stuttgart, 1987 (coll. « Corpus Cosmatorum », 1) ; X. BARRAL I ALTET, Els mosaïcs de paviment medievals a Catalunya, Barcelone, 1979 (coll. " Art romànic », 10). Nous remercions Géraldine Mallet, maître de conférences, université de Montpellier, d'avoir attiré notre attention sur ces remplois.

33. X. BARRAl I Altet, Les mosaïques de pavement médiévales de Venise, Murano/Torcello/Paris, 2000. 
34. F. Guidobaldi et A. GUiglia Guidobaldi, Pavimenti marmorei di Roma dal IV al IX secolo, Cité du Vatican, 1983.

35. R. DONDARINI (dir.), Farfa, abbazia imperiale, Negarine, 2006 ; concernant le pavement de Castel S. Elia, voir : http://matematica.unibocconi.it/articoli/i-pavimenti-dei-cosmati.

36. Ce matériel a été étudié par J. Rollier-Hanselmann, dans le cadre du Corpus de la sculpture de Cluny..., op. cit., t. 1.

37. Gilles Rollier, fouilles de l'hiver 1989-1990. Lors des fouilles d'Anne Baud et de Christian Sapin, en 2006-2007 (partie précédant le chevet de Cluny II), de nouveaux fragments sont apparus.

38. Analyses préliminaires effectuées sur le bleu par le Professeur Corrado Gratziu de l'université de Pise.

39. N. STRATFORD et alii, Corpus de la sculpture de Cluny ..., op. cit.

40. Les références du document sont dans le Corpus de la sculpture de Cluny..., ibid.

41. Laurent Guyot (1756-1806) travailla pour Lenoir. Il fit des reproductions d'art pour le nouveau musée des Monuments français.

42. Inventaire général des richesses d'art de la France. Archives du musée des Monuments français. $1^{\text {re }}$ partie. Papiers de M. Albert Lenoir, Paris, 1883, p. 179-182 ; K. J. ConANT, Cluny..., op. cit., p. 11 ; N. STRATFORD et alli, Corpus de la sculpture de Cluny..., op. cit., t. 1.

43. M. P. LORAIN, Essai historique sur l'abbaye de Cluny, 1839, p. 86 cité par F. MERCIER, Les primitifs français. La peinture clunysienne en Bourgogne à l'époque romane : son histoire et sa technique, Paris, 1931, p. 87.

44. K. J. CONANT, Cluny..., op. cit., p. 16.

45. Lettre de M. Furtin, maire de Cluny, octobre 1811, Archives de Cluny, F II, casier 42 (P5). N. STRATFORD, « Visite de Berzé-la-Ville », in Le gouvernement d'Hugues de Semur, 1988, note 29.

46. J. ROLLIER-HANSELMANN, « D'Auxerre à Cluny : technique de la peinture murale entre le $\mathrm{VIII}^{\mathrm{e}}$ et le XII ${ }^{\mathrm{e}}$ siècle en Bourgogne », Cahiers de civilisation médiévale, 40 (1997), p. 57-90.

47. Y. CHRISTE, «À propos de deux découvertes récentes : les peintures de la cathédrale de Nevers et de Saint-Sylvain de Chalivoy-Milon », Cahiers archéologiques, 38 (1990), p. 91-98; M. ANGHEBEN, Les chapiteaux romans de Bourgogne, Turnhout, 2003, p. 33-38.

48. J. ROLliER-HANSELMANN et S. CASTANDET, «Couleurs et dorures du portail roman de Cluny III. Restitution en 3D d'une œuvre disparue», Bucema, 14 (2010), p. 235-250 [http://cem.revues.org/ index11601.html].

49. C. Di matteo et P.-A. LABLAUdE, « Notre-Dame d'Étampes ", Monuments historiques (161) 1989, p. 86-90. Le portail sud est daté vers 1130-1140. Des dorures ont été découvertes dans l'ancien palais abbatial de Moissac, de datation incertaine. S. CASTANDET, Les décors métalliques dans les peintures murales ( $\mathrm{XI}^{e}$-XVII ${ }^{e}$ siècle). Technique et altération, mémoire de master 2 , université de Bordeaux 3, 2008.

50. F. MERCIER, Les primitifs français..., op. cit., p. 87.

51. J.R Ollier-hanselmann, "Étude technique des fragments de peintures murales et des polychromies sur pierre provenant de l'abbaye de Cluny ( $\mathrm{XII}^{\mathrm{e}}-\mathrm{XV}^{\mathrm{e}}$ siècle) ", in N. STRATFORD et alii,Corpus de la sculpture de Cluny..., op. cit., t. 1 .

52. A. BAUD, Cluny, un grand chantier médiéval au cour de l'Europe, Paris, 2003, p. 127.

53. B. DUMOLIN, Description historique..., op. cit.

54. A. BAUD, G. ROLlier et G. MACABEO, Le transept sud de l'abbaye de Cluny, DFS de sauvetage programmé (non publié), 4 vol., Dijon, 1994.

55. Éléments présents dans les US 172, 1001, US 1003 et US 1005.

56. A. BAUD, Le chantier de la troisième église abbatiale de Cluny, thèse de doctorat, université de Lyon, 1996 ; A. BAUD, G. ROLLIER et G. MACABEO, Le transept sud..., op. cit. 
57. H. ERISTOV et S. GROETEMBRIL, «Une fenêtre peinte ? ", in De transparentes spéculations. Vitres de l'Antiquité et du haut Moyen Âge (Occident-Orient), Bavay, 2005, p. 29.

58. Analyses effectuées par S. Castandet au laboratoire CRP2A de l'université de Bordeaux 3. Travaux en cours de publication par G. Rollier.

59. L. GRODECKI, Le vitrail roman, Fribourg, 1977, p. 286.

60. Comparaisons avec les manuscrits clunisiens, tels que le Lectionnaire de Cluny, et les peintures murales de la Chapelle-des-Moines, à Berzé-la-Ville.

INDEX

Index géographique : France/Cluny

Mots-clés : clôture de chœur, Cluny III, pavement, peinture murale, reconstitution 3D, stalle, vitrail 